\title{
DESAFÍOS DEL APRENDIZAJE DIGITAL PARA LA FORMULACIÓN DE PROYECTOS PEDAGOGICOS EN LA AGROINDUSTRIA
}

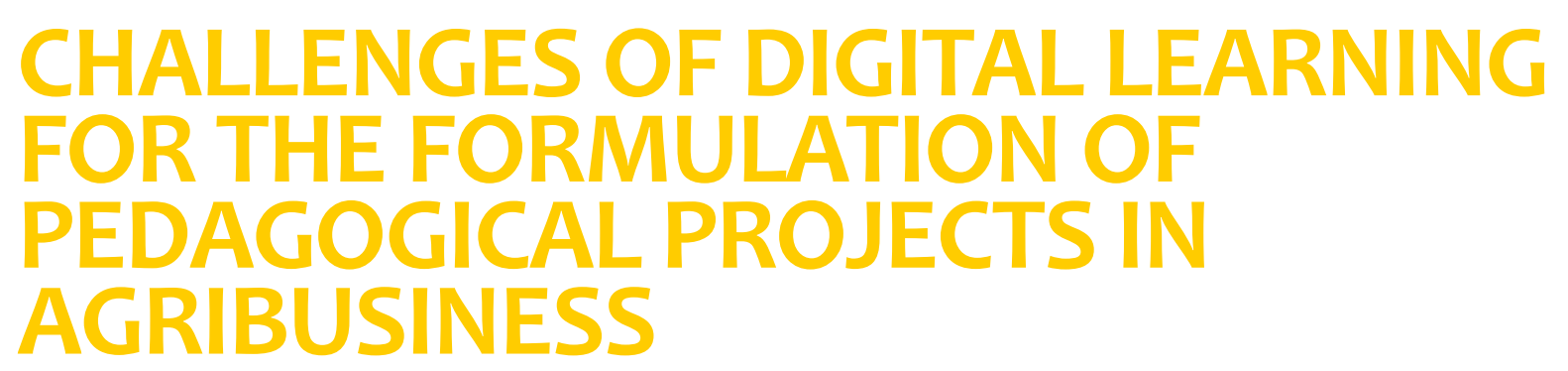

Jesús Alfonso Torres Ortega ${ }^{1^{*}}$

Oscar Fernando Contento Rubio ${ }^{2}$

Universidad La Salle

\section{RESUMEN}

Plantear soluciones adecuadas para contribuir en la construcción de conocimiento para el agro es una meta incluyente no sólo para las entidades gubernamentales sino un compromiso de las instituciones educativas involucradas con el campo. Para lograr este fin que busca la equidad en el campo y además mejorar la innovación para los agricultores, es primordial atender las brechas que hay entre el campo y la ciudad, y hacer que la sistematización llegue a sus manos de forma práctica y sencilla. Sin embargo, ¿Qué medidas toma el agro para la cuarta revolución industrial? Es forzoso refinar

1* Ing. Químico, MSc, Doctor en Ingeniería, Profesor Asociado, Programa de Ingeniería Ambiental y Sanitaria, Facultad de Ingeniería, Universidad de La Salle. Carrera 2 No. 10-70, Bogotá, Colombia. Teléfono: (+571) 3535360 extensión: 2515, email: jatorres@unisalle.edu.co; http://orcid.org/0000-0002-4476-0816; https://scholar.google.es/citations?hl=es\&user=t5LjNycAAAAJ 
recursos educativos digitales orientados a apoyar las actividades agroindustriales en el campo empleando las herramientas que brindan las Tecnologías de la Información y la Comunicación (TICs) bajo la perspectiva de una economía circular.

Con las TICs se busca promover el liderazgo de nuestros habitantes rurales, a través de procesos de capacitación pertinente al alcance de todos, orientados siempre al campo y que busquen una apropiación del campesino, su inclusión y desarrollo, ya que estas herramientas aprovechadas en los trabajos del campo son la principal motivación en la búsqueda de la alfabetización digital que permita alcanzar un conocimiento adecuado de su entorno y la adaptación al cambio climático.

PALABRAS CLAVE: Agrícola, formación, industria, información, tecnología.

\section{ABSTRACT}

Proposing adequate solutions to contribute to the construction of knowledge for agriculture is an inclusive goal not only for government entities but a commitment of the educational institutions involved with the field. To achieve this end, which seeks equity in the countryside and also to improve innovation for farmers, it is essential to address the gaps between the countryside and the city, and make systematization reach their hands in a practical and simple way. However, what measures does agriculture take for the fourth industrial revolution? It is necessary to refine digital educational resources aimed at supporting agro-industrial activities in the field using the tools that Information and Communication Technologies (ICTs) provide under the perspective of a circular economy. ICTs seek to promote the leadership of our rural inhabitants, through relevant training processes available to all, always oriented to the field and seeking the appropriation of the farmer, their inclusion and development, since these tools used in the work from the field are the main motivation in the search for digital literacy that allows achieving adequate knowledge of their environment and adaptation to climate change.

KEYWORDS: Agricultural, industry, information, technology, training.

\section{INTRODUCCIÓN}

El año 2020 empieza con 4.500 millones de internautas en el planeta con acceso al internet, y se espera que en la próxima década, el número escale rápidamente a 6.000 millones, quienes con la llegada de la tecnología de conectividad $5 G$, podrán navegar a velocidades hasta 10 veces mayores que la rutina más rápida actual. Con el cambio de siglo el internet de ultra velocidad abarca los aspectos más diversos de nuestras vidas, la incidencia de su impacto en nuestra salud y costumbres es el centro de una concentración creciente de investigaciones que señalan sobre la necesidad de reflexionar y generar un escenario accesible y comprensible (Rúa, 2019).

Al evaluar las condiciones técnicas de las prácticas agroindustriales, con los impactos ambientales, positivos o negativos, bajo la óptica de las ciencias agrarias, la ruralidad o la bioeconomía; surgen algunos cuestionamientos tanto metodológicos, como procedimentales, sistemáticos, tecnológicos, políticos o incluso éticos (Giraldo, 2019). La relación con la asignación valorativa que estos impactos puedan llegar a tener, que en general demandan de un estudio integral, para involucrar todos los factores relacionados con la manufactura, la innovación y la sostenibilidad en el ciclo productivo e identificar los procesos comprometidos en el aprendizaje para diseñar estrategias adecuadas para la socialización de las herramientas digitales contemporáneas y que son de uso común en el primer mundo. 
¿Es posible plantear soluciones adecuadas para contribuir en la construcción de conocimiento para el agro apoyados en el diseño y gestión de escenarios virtuales de aprendizaje para los agricultores? Autoridades de educación revisan la formación de investigadores y la transformación digital con el fin de impulsar la innovación en Iberoamérica y colocarla al servicio de la verificación del cumplimiento de los objetivos de Desarrollo Sostenible (SEGIB, 2020). Es necesario ampliar el concepto a la condición multidisciplinar en donde se pueda comunicar de manera eficiente y asertiva las nuevas tecnologías aplicadas al agro y que mejor escenario para el alcance de esta meta que diseñar proyectos educativos que permitan evaluar las estrategias adecuadas para la promoción de escenarios virtuales para consolidar la comunicación en la universidad frente a los desafíos de la sociedad red.

\section{CONTEXTO}

Colombia cuenta con más de 40 millones de hectáreas reconocidas para cosechar productos que garanticen la seguridad alimentaria, sin embargo las cosechas se hacen en solo 7 millones de hectáreas y se importa gran cantidad de los alimentos que tienen potencial en nuestros pisos térmicos (PND, 2018-2022). A esto se suma los riesgos que implica el cambio climático y la dificultad que tienen los agricultores para obtener ganancias de sus siembras, debido a que el país solo cuenta con 175.000 kilómetros de vías terciarias no les es fácil sacar sus productos, sumado al $86 \%$ de la informalidad laboral que hay en el campo (SAC, 2019). Las Tecnologías de la Información y la Comunicación (TICs) aplicadas a las actividades económicas del campesino son la motivación principal en la búsqueda de la alfabetización digital, conocimiento del medio, adaptación al cambio climático, en su componente didáctico para huertas caseras, cultivos agroenergéticos o incluso en la formación de proyecto de vida para jóvenes. Con las TICs se busca promover el liderazgo de nuestros habitantes rurales, a través de procesos de enseñanza adecuada, dirigidos siempre al campo y que busquen la dignificación del campesino, su inclusión y desarrollo (Lavacude, 2019).

De otra parte, para ayudar a los países que tienen problemas para cumplir sus metas de reducción de gases de efecto de invernadero (GEI), convertidas en emisiones de $\mathrm{CO}_{2}$ en algunos sectores, se crearon los mecanismos del mercado internacional de carbono y así ayudar a cumplir la meta de mantener la temperatura global terrestre por debajo de $2^{\circ} \mathrm{C}$. Uno de los temas más complejos y polémicos que se debate desde la Conferencia de las Partes (COP25) en Madrid (2019). Con el Acuerdo de París, firmado en 2015, Colombia se comprometió a reducir un $20 \%$ para el 2030 con inversión nacional y $30 \%$ con cooperación internacional. Sin embargo, en muchas zonas, la infraestructura es el factor crítico, ya que los habitantes de la ruralidad se encuentran rezagados digitalmente lo que conlleva a que el agricultor reciba bajas ganancias por su producción (PND, 2018-2022).

Muchos proyectos de investigación están apuntando al fortalecimiento de la competitividad del sector agrícola mediante el uso de ciencia, tecnología e innovación para el aprovechamiento de los residuos de los cultivos (Wang et al., 2019; Liu et al., 2016). Existen tecnologías digitales, probadas y disponibles comercialmente que requieren de optimización e implementación en puntos específicos del territorio nacional que permitirán el desarrollo de la región. Al optimizar la cadena productiva del agro colombiano, se logra aprovechar residuos agrícolas industriales, con poco o nulo uso, disminuyendo impactos ambientales con el reúso de residuos potencialmente contaminantes $\mathrm{o}$ con altos costos de disposición, además al disminuir gradualmente el consumo de combustibles provenientes del petróleo, se reduce la emisión 
de $\mathrm{CO}_{2}$ y otros contaminantes; adicionalmente es posible que la optimización del ciclo productivo genere empleo en cada etapa, favoreciendo la mano de obra no calificada y los sectores rurales, impulsando el crecimiento del sector agrícola (Duque et al., 2020; Mouratiadou et al., 2020).

Por otra parte, la literatura científica permite establecer el concepto de 'seguridad alimentaria' en términos de la distribución de los excedentes de la producción agrícola, esta perspectiva conduce a la necesidad de identificar las relaciones de equidad y comercio justo, necesarios para garantizar el desarrollo de prácticas agrícolas sustentables. Entonces, sugerir aspectos relevantes para la formulación de tecnologías emergentes con enfoque diferenciado, mediante la integración de actores nuevos de valorización de los excedentes, usos del bioresiduo, el acceso a recursos y know-how, conlleva a una estructuración de las cadenas de valor (Pathak y Chandel, 2017).

\section{ANTECEDENTES}

El colombiano promedio usa las nuevas tecnologías digitales comúnmente para consultar redes sociales y buscar información, y menos con fines educativos y aplicaciones industriales. Las redes más usadas son Facebook y Whatsapp, con más de 18 millones de usuarios y la cifra crece exponencialmente, mientras los usuarios de las aplicaciones de banca móvil llegan a 3,5 millones, según BBVA Research. Por el contrario aplicaciones para teléfonos móviles como PlantSnap (plant.net) ó PlantNet (planet. org) para identificar plantas, flores y más, son poco utilizadas o desconocidas y que cuentan con un gran inventario de plantas.

Las condiciones ambientales, en particular lo relacionado con las emisiones de gases que contribuyen al efecto de invernadero, la disposición de residuos agroindustriales, la sostenibilidad agraria y sostenibilidad de la cadena de producción promueven el mejoramiento de tecnologías que utilizan fuentes renovables de energía, dentro de las cuales se encuentra la valorización de los residuos agrícolas, la optimización de procesos industriales juega un papel importante para el aprovechamiento de los residuos orgánicos de la agroindustria y la disminución de impactos ambientales asociados. 'Misión 1.5' es un video juego para teléfonos móviles es una novedosa forma de las Naciones Unidas para conocer las opiniones de los ciudadanos para decidir que medidas adoptar frente al calentamiento global (PNUD, 2020)

Un punto importante a atender en el agro es reducir la volatilidad de los precios y aumentar el rendimiento de la producción a través de su ordenamiento. En diciembre de 2018, se lanzó oficialmente "Coseche y venda a la fija", un programa que asegura la comercialización de los productos agropecuarios a los campesinos del país a través de los contratos de venta anticipada. Colombia recibirá 360.000 millones para reducir la deforestación en 155.000 hectáreas para el 2022 y 155.000 en 2025. Por otro lado, para el 2022 se incluirán 195.000 hectáreas adicionales bajo el programa de pago por servicios ambientales, incluyendo tierras ubicadas en territorios colectivos de comunidades étnicas. Para lograr esto se generarán plantaciones forestales por 122.000 hectáreas, se renovarán otras 70.000 de cacao envejecido en el 2022, y se aumentarán 40.000 las hectáreas de algodón. Como se aprecia en la Figura 1 las flores son un renglón muy importante, siendo el departamento de Cundinamarca la región con mayor participación en el mercado nacional (Farfán, 2019).

Un caso particular lo constituye la palma de aceite; en el país hay 450.131 hectáreas cultivadas con palma de aceite y a que los rendimientos de los cultivos comerciales de palmas $E$. guineensis adultas oscilan entre 21 
y 30 toneladas de racimos de fruta fresca (RFF) por hectárea, mientras que el correspondiente a los cultivos $O x G$ oscila entre 22 y 32 toneladas de RFF por hectárea, el costo promedio por tonelada de (RFF) en 2014 era US\$ 247.181 para cultivos de E. guineensis, el costo por tonelada de aceite de palma crudo (ACP) fue US\$ 1.365.607 para cultivos de E. guineensis (Franco et al., 2019).
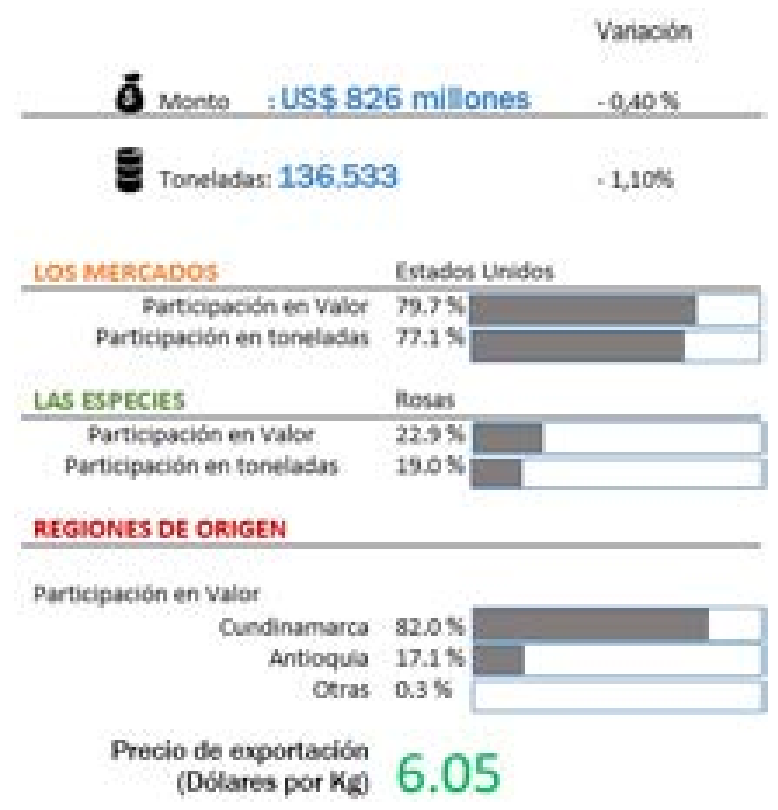

Fuente: Asocolflores.

Figura 1. Algunas cifras del sector exportador de flores Enero - Junio 2019. Tomado de: El Tiempo. Economía: Solo el $5 \%$ de la producción de flores se vende en el país. Viernes 20 de septiembre de 2019, pág. 1.8 .

En relación con la condición ambiental, un indicador ambiental que se ha utilizado en las últimas décadas es la huella hídrica generada por la producción de este sector que asciende a unos 2.000 metros cúbicos por tonelada de palma de fruta, entendido este indicador como el gasto del recurso hídricos (agua virtual incluido) para la producción del producto en específico, lo anterior llama la atención por la posibilidad de crear un problema de seguridad alimentaria y la disponibilidad del recurso agua para las generaciones futuras. Muchas aplicaciones se encuentran en el mercado que permiten una estimación de este indicador para el agro, CropWat, ArcGIS y SimaPro son muy utilizados en el agro, en especial este último por las bases de datos entre la que destaca Ecolnvent (Corba et al., 2018; Fonseca, 2016; Vanegas, 2014).

\section{VIRTUALIDAD}

Existen tecnologías digitales, probadas y disponibles comercialmente que requieren de optimización e implementación en puntos específicos del territorio nacional que permitirán el desarrollo de la región. Al optimizar la cadena productiva del agro colombiano, se logra aprovechar residuos agrícolas industriales, con poco o nulo uso, disminuyendo impactos ambientales con la reutilización de los residuos potencialmente contaminantes y con altos costos de disposición, además al disminuir gradualmente el consumo de combustibles provenientes del petróleo, se reduce la emisión de $\mathrm{CO}_{2}$ y otros contaminantes; adicionalmente es posible que la optimización de la ciclo productivo genere empleo en cada etapa, favoreciendo la mano de obra no calificada y los sectores rurales, impulsando el crecimiento del sector agrícola (Duque et al., 2020).

Una conclusión a la que llegaron expertos internacionales que buscaban definir como será la educación en la próxima década, encontró que, en los próximos 15 años, internet va a convertir los centros educativos en "entornos interactivos", estos revolucionarán las formas tradicionales de aprendizaje y cambiarán las formas pedagógicas de enseñar. El informe ha sido realizado por la Cumbre Mundial para la Innovación en Educación (WISE por su acrónimo en inglés). En Colombia seguimos muy pegados a una visión operativa de la prestación de los servicios educativos, como si con la mera eficiencia del sistema y la leve mejora en los resultados académicos fuera suficiente para el progreso del país. Hoy se habla de sistemas ciberfísicos que combinan maquinaria 
con procesos digitales y son capaces de tomar decisiones descentralizadas y de cooperar entre ellos y con los humanos - mediante el internet de las cosas.

Estas transformaciones están modificando el mundo del trabajo, como lo hicieron las computadoras hace 25 años (Caijao, 2019). Lo que importa es que semejantes progresos solo beneficiarán a quienes sean capaces de innovar y adaptarse. La brecha no va en la tecnología. Ella va a llegar, los datos pueden producirse y la innovación se dará en todas partes. La brecha estará en el sistema educativo (upskilling digital). Esta información no tiene que ver con un usuario, sino con máquinas, compañías, granjas, fábricas, etc. Se trata de datos para administrar negocios de una forma diferente, dado que lo que se conecta con la cuarta revolución industrial son drones, máquinas y dispositivos loT (Internet of Things), se esta creando una base de datos totalmente nueva que nos permiten conocer más de las cosas y serán tan fácil como conectarse a un celular (Kande, 2019).

El Observatorio de la Universidad Colombiana (2020) plantea que de las crisis vienen oportunidades, y la virtualidad es una de ellas. Ante una situación como una epidemia la suspensión de actividad académica ante un posible contagio, crea alarma sobre la responsabilidad de las Instituciones de Educación Superior (IES) en la conducción de la situación. En el campo con mucha regularidad se está expuesto a tragedias naturales que impiden la movilización entre las veredas. Claramente la mejor forma de evitar la proliferación de la enfermedad ya que las reuniones de personas es un mecanismo de contagio. Por ello, la opción de la virtualidad, se establece como una oportunidad que cada día alcanza más demanda por la practicidad para eliminar las barreras físicas. Cada persona, desde su propio espacio, enfermo o no, puede adelantar su estudio, en sus tiempos, sin tener que verse incomodado por restricciones o recomendaciones de privación en desplazamientos y movimientos.

\section{CONCLUSIÓN}

Diseñar recursos digitales de acuerdo con las fases de un proceso de producción para el alcance de una finalidad formativa, orientada a impulsar las gestiones del campo, contribuirá a estimular la conectividad en el agro colombiano. Estos instrumentos han motivado la creación de indicadores que permitan una administración eficiente de los recursos disponibles, que facilitan cuantificar los insumos requeridos para generar bienes y servicios disminuyendo sus impactos ambientales desde una concepción multidisciplinar por cuanto las TICs se pueden constituir en una herramienta primordial en la búsqueda de la alfabetización digital que permita alcanzar al agricultor una comprensión adecuada de su entorno y lo prepara en la adaptación al cambio climático.

\section{AGRADECIMIENTO}

Los autores agradecen el apoyo de la investigación: "Aprovechamiento de residuos derivados de la producción de flores ornamentales en la sabana de Bogotá como materia prima de una biorefinería piloto", por la financiación cuyo número de departamento asignado al Proyecto VRIT 2018 es 243286.

\section{REFERENCIAS BIBLIOGRÁFICAS}

Caijao F. (2019). Las redes de la educación. Tomado de: El Tiempo. Las claves 2020 2030. Domingo 15 de diciembre de 2019, pág. 5.6.

Corba J., Vargas L. y Torres J. (2018). Determinación de la huella hídrica para un cultivo de guayaba pera (Psidium guajava). Revista Publicaciones e Investigación. Bogotá, Colombia, Vol. 12(2): 69-81. 
Duque M., Fuquene D. y Torres J. (2020). Estimación de la huella de carbono por medio del software SimaPro para cultivos de plantas ornamentales en una empresa de Colviveros con base en los lineamientos de la norma ISO 14044. Tesis. Universidad de La Salle, Colombia.

Farfán E. (2019). Solo el 5\% de la producción de flores se vende en el país. Tomado de: El Tiempo. Economía. Viernes 20 de septiembre de 2019, pág. 1.8 .

Fedepalma (2015). Anuario Estadístico 2015. La agroindustria del aceite de palma en Colombia y el mundo 2010 - 2014. Bogotá. Colombia.

Fonseca O., Lizarazo J. y Torres J. (2016). Determinación de la huella hídrica del algodón. Revista Publicaciones e Investigación. Bogotá, Colombia, Vol. 10: 3946.

Franco J., Ordoñez L., Herrera I. y Torres J. (2019). Análisis de ciclo de vida para la producción de biodiesel derivado de palma de aceite, caso colombiano. Revista Publicaciones e Investigación. Bogotá, Colombia, Vol. 13: 11-26.

Giraldo J. (2019). El papel de la nueva ruralidad en el desarrollo rural integral, una vista comparativa entre la Republica Popular China y la Republica de Colombia. Tesis. Universidad de Ciencias Aplicadas y Ambientales, Colombia.

Guerrero A. (2019). Mercados de carbono, cuello de botella de la COP25. Tomado de: El Tiempo. Medioambiente. Martes 10 de diciembre de 2019. Recuperado de: https:// www.eltiempo.com/vida/medio-ambiente/ mercados-de-carbono-el-cuello-de-botellade-la-cop25-442184

Kande M. (2019). La innovación con tecnología no depende del dinero. Tomado de: El Tiempo. Innova 2.0. Domingo 27 de octubre de 2019, pág. 6.14.

Liu, Z., Liao, W., Liu, Y. (2016). A sustainable biorefinery to convert agricultural residues into value-added chemicals. Biotechnology for Biofuels, 9 (1), art. no. 197.

Lavacude K. (2019). Manos amigas buscan el progreso del campo. Tomado de: El Tiempo. Informe sectorial. Miércoles 30 de octubre de 2019, pág. 7.

Mouratiadou, I., Stella, T., Gaiser, T., Wicke, B., Nendel, C., Ewert, F., van der Hilst, F. (2020). Sustainable intensification of crop residue exploitation for bioenergy: Opportunities and challenges. GCB Bioenergy, 12 (1), pp. 7189.

Observatorio de la Universidad Colombiana (2020). Virtualidad, una oportunidad para las IES en época de Coronavirus. Tomado de: https://www.universidad.edu.co/virtualidaduna-oportunidad-para-la-ies-en-epoca-decoronavirus/

Pathak, B.S., Chandel, A.K. (2017). Feedstock transportation, agricultural processing, logistic from farm to bio-refinery: Recent developments, mechanization, and cost analysis Sustainable Biofuels Development in India, pp. 207-221.

Plan Nacional de Desarrollo - PND 2018 2022. Recuperado de https://www.dnp. gov.co/DNPN/Paginas/Plan-Nacional-deDesarrollo.aspx

Programa de Naciones Unidas para el Desarrollo - PNUD. (2020). Un video juego sobre el cambio climático. Tomado de: ADN. EFE, Nueva York, USA. 14 de febrero de 2020, pág. 21. 
Rúa M. (2019). En busca del bienestar digital, una preocupación ya necesaria. El Tiempo. 27 de octubre de 2019, pág. 2.7 .

Sánchez, G. (2009). Análisis de la sostenibilidad agraria mediante indicadores sintéticos: aplicación empírica para sistemas agrarios de Castilla y León. Tesis. Universidad Politécnica de Madrid, España.

Secretaría General Iberoamericana - SEGIB. (2020). Los retos de la $U$ en Iberoamérica. Tomado de: ADN. EFE, La Habana. Cuba. 11 de febrero de 2020, pág. 8.

Sociedad de Agricultores de Colombia (SAC). 2019. 39 Congreso Agropecuario Nacional. 6-7 de noviembre de 2019. Bogotá. Recuperado de https://sac.org.co/.

Torres J, Contento O., Orozco I, (2017). Análisis de ciclo de vida para una biorefinería derivada de residuos agrícolas de palma aceitera (Elaeis Guineensis). Revista Publicaciones e Investigación. Bogotá, Colombia, Vol. 11: 13-36.
Torres J., Herrera I, Garrain D., Gamarra A. (2018). Integration of a Colombian biorefinery from industrial palm oil waste into the circular economy. Abstract Book, SETAC Europe 28th Annual Meeting, 13 - 17 May 2018, Rome, Italy, pp. 289.

Valencia A. (2019). Las grandes apuestas que vienen para el agro. Tomado de: El Tiempo. Especial. Domingo 8 de diciembre de 2019, pág. 1.33.

Vanegas Y., Vera L. y Torres J. (2014). Evaluación de la huella hídrica del lirio japonés. Revista Publicaciones e Investigación. Bogotá, Colombia, Vol. 8: 79-87.

Wang, Y., Ke, L., Yang, Q., Peng, Y., Hu, Y., Dai, L., Jiang, L., Wu, Q., Liu, Y., Ruan, R., Fu, G. (2019). Biorefinery process for production of bioactive compounds and biooil from Camellia oleifera shell. International Journal of Agricultural and Biological Engineering, 12 (5), pp. 190-194. 